\title{
TU/e EmonOWEN

\section{A generic approach to the filtering of matrix fields with singular PDEs}

\section{Citation for published version (APA):}

Burgeth, B., Didas, S., Florack, L., \& Weickert, J. (2007). A generic approach to the filtering of matrix fields with singular PDEs. In F. Sgallari, A. Murli, \& N. Paragios (Eds.), Proceedings of the First International Conference on Scale Space and Variational Methods in Computer Vision (SSVM 2007) 30 May - 2 June 2007, Ischia, Italy (pp. 556-567). (Lecture Notes in Computer Science; Vol. 4485). Springer. https://doi.org/10.1007/978-3-54072823-8_48

DOI:

10.1007/978-3-540-72823-8_48

Document status and date:

Published: 01/01/2007

\section{Document Version:}

Publisher's PDF, also known as Version of Record (includes final page, issue and volume numbers)

\section{Please check the document version of this publication:}

- A submitted manuscript is the version of the article upon submission and before peer-review. There can be important differences between the submitted version and the official published version of record. People interested in the research are advised to contact the author for the final version of the publication, or visit the $\mathrm{DOI}$ to the publisher's website.

- The final author version and the galley proof are versions of the publication after peer review.

- The final published version features the final layout of the paper including the volume, issue and page numbers.

Link to publication

\section{General rights}

Copyright and moral rights for the publications made accessible in the public portal are retained by the authors and/or other copyright owners and it is a condition of accessing publications that users recognise and abide by the legal requirements associated with these rights.

- Users may download and print one copy of any publication from the public portal for the purpose of private study or research.

- You may not further distribute the material or use it for any profit-making activity or commercial gain

- You may freely distribute the URL identifying the publication in the public portal.

If the publication is distributed under the terms of Article $25 \mathrm{fa}$ of the Dutch Copyright Act, indicated by the "Taverne" license above, please follow below link for the End User Agreement:

www.tue.nl/taverne

Take down policy

If you believe that this document breaches copyright please contact us at:

openaccess@tue.nl

providing details and we will investigate your claim. 


\title{
A Generic Approach to the Filtering of Matrix Fields with Singular PDEs
}

\author{
Bernhard Burgeth ${ }^{\star}$, Stephan Didas ${ }^{\star \star}$, Luc Florack ${ }^{\star}$, and Joachim Weickert \\ Saarland University, Dept. of Mathematics and Computer Science, \\ Germany \\ burgeth@mia.uni-saarland.de \\ Eindhoven University of Technology, Dept. of Biomedical Engineering \\ The Netherlands \\ L.M.Florack@tue.nl
}

\begin{abstract}
There is an increasing demand to develop image processing tools for the filtering and analysis of matrix-valued data, so-called matrix fields. In the case of scalar-valued images parabolic partial differential equations (PDEs) are widely used to perform filtering and denoising processes. Especially interesting from a theoretical as well as from a practical point of view are PDEs with singular diffusivities describing processes like total variation (TV-)diffusion, mean curvature motion and its generalisation, the so-called self-snakes. In this contribution we propose a generic framework that allows us to find the matrix-valued counterparts of the equations mentioned above. In order to solve these novel matrix-valued PDEs successfully we develop truly matrix-valued analogs to numerical solution schemes of the scalar setting. Numerical experiments performed on both synthetic and real world data substantiate the effectiveness of our matrix-valued, singular diffusion filters.
\end{abstract}

\section{Introduction}

Matrix-fields are used, for instance, in civil engineering to describe anistropic behaviour of physical quantities. Stress and diffusion tensors are prominent examples. The output of diffusion tensor magnetic resonance imaging (DT-MRI) 14 are symmetric $3 \times 3$-matrix fields as well. In medical sciences this image acquisition technique has become an indispensable diagnostic tool in recent years. Evidently there is an increasing demand to develop image processing tools for the filtering and analysis of such matrix-valued data.

d-dimensional scalar images $f: \Omega \subset \mathbb{R}^{d} \rightarrow \mathbb{R}$ have been denoised, segmented and/or enhanced successfully with various filters described by nonlinear parabolic PDEs. In this article we focus on some prominent examples of PDEs used in image processing and which can serve as a proof-of-concept:

\footnotetext{
* The financial support of the Dutch Organization for Scientific Research NWO is gratefully acknowledged.

** The financial support of the German Organization for Scientific Research DFG is gratefully acknowledged.
} 
- Total-Variation (TV)-Diffusion ( $\mathrm{p}=1$ ), [3]10] and balanced-forward-backward (BFB)-diffusion $(\mathrm{p}=2)$, [13],

$$
\partial_{t} u=\operatorname{div}\left(\frac{\nabla u}{\|\nabla u\|^{p}}\right),
$$

- Mean curvature motion (MCM), [2],

$$
\partial_{t} u=\|\nabla u\| \operatorname{div}\left(\frac{\nabla u}{\|\nabla u\|}\right),
$$

- Self-Snakes involving a Perona-Malik type diffusivity $g$, [15,

$$
\partial_{t} u=\|\nabla u\| \operatorname{div}\left(g\left(\|\nabla u\|^{2}\right) \frac{\nabla u}{\|\nabla u\|}\right),
$$

where we impose the initial condition $u(x, 0)=f(x)$ for $x \in \Omega$ in all cases. TV-type diffusion filters require no tuning of parameters but have shapepreserving qualities [6] and a finite extinction time [4]. Even arbitrary exponents have been considered, 1117. Extensions of curvature-based PDEs to matrix fields have been proposed in [11 and more recently in [16, based on generalisations of the so-called structure tensor for scalar images to matrix fields. The research on these structure-tensor concepts has been initiated by $7 / 19$. The approaches to matrix field regularisation suggested in 9 are based on differential geometric considerations. Comprehensive survey articles on the analysis of matrix fields using various techniques can be found in 20 .

In this article we will proceed along a different path. We will develop a generic framework for deriving matrix-valued counterparts for scalar PDEs. This does not just mean that we derive systems of PDEs which can be written in matrix form. Instead we will exploit the operator-algebraic properties of (symmetric) matrices to establish truly matrix-valued PDEs. For this work we concentrate on the matrix-valued analogs of the singular PDEs (10)-(3) as particularly interesting equations. It is also worth mentioning that in contrast to [11] and [16] our framework does not rely on a notion of structure tensor. Nevertheless, the proposed concept ensures an appropriate and desirable coupling of channels. The methodology to be developed will also enable us to transfer numerical schemes from the scalar to the matrix valued setting.

The article is structured as follows: The subsequent Section 2 contains the basic definitions necessary for our framework, such as functions of a matrix, partial derivatives, and generalised gradient of a matrix field. In Section 3 we turn first to the simple linear diffusion for matrix fields for the sake of later comparison. After introducing a symmetrised multiplication for symmetric matrices we then formulate the matrix-valued counterparts of the singular equations mentioned above. By considering the already rather complicated one-dimensional case, first properties of the matrix-valued diffusion processes are inferred. The transition from scalar numerical solution schemes to matrix-valued algorithms for the solutions of the new diffusion equations is discussed in Section 4 Example applications on synthetic and real DT-MRI data are presented in Section 5 , followed by concluding remarks in the last Section 6 . 


\section{Matrix-Valued PDEs: A Generic Framework}

This section contains the key definitions for the formulation of matrix-valued PDEs. The underlying idea is that to a certain extent symmetric matrices can be regarded as a generalisation of real numbers. In that spirit we would like to generalise notions like functions of matrices, derivatives and gradients of such functions to the matrix-valued setting as instigated in 8 . We juxtapose the corresponding basic definitions in Table 1 , and comment on them in the subsequent remarks. We start with clarifying notation. A matrix field is considered as a mapping $F: \Omega \subset \mathbb{R}^{d} \longrightarrow M_{n}(\mathbb{R})$, from a $d$-dimensional image domain into the set of $n \times n$-matrices with real entries, $F(x)=\left(f_{p, q}(x)\right)_{p, q=1, \ldots, n}$. Important for us is the subset of symmetric matrices $\operatorname{Sym}_{n}(\mathbb{R})$. The set of positive (semi-) definite matrices, denoted by $\operatorname{Sym}_{n}^{++}(\mathbb{R})\left(\operatorname{resp} ., \operatorname{Sym}_{n}^{+}(\mathbb{R})\right)$, consists of all symmetric matrices $A$ with $\langle v, A v\rangle:=v^{\top} A v>0 \quad$ (resp., $\geq 0$ ) for $v \in \mathbb{R}^{n} \backslash\{0\}$. This set is of special interest since DT-MRI produces data with this property. Note that at each point the matrix $F(x)$ of a field of symmetric matrices can be diagonalised yielding $F(x)=V(x)^{\top} D(x) V(x)$, where $x \mapsto V(x) \in O(n)$ is a

Table 1. Extensions of elements of scalar valued calculus (middle) to the matrixvalued setting (right)

\begin{tabular}{|c|c|c|}
\hline Setting & scalar valued & matrix-valued \\
\hline function & $h:\left\{\begin{array}{l}\mathbb{R} \longrightarrow \mathbb{R} \\
x \mapsto h(x)\end{array}\right.$ & $h:\left\{\begin{array}{l}\operatorname{Sym}_{n}(\mathbb{R}) \longrightarrow \operatorname{Sym}_{n}(\mathbb{R}) \\
U \mapsto V^{\top} \operatorname{diag}\left(h\left(\lambda_{1}\right), \ldots, h\left(\lambda_{n}\right)\right) V\end{array}\right.$ \\
\hline $\begin{array}{c}\text { partial } \\
\text { derivatives }\end{array}$ & $\begin{array}{c}\partial_{\omega} u \\
\omega \in\left\{t, x_{1}, \ldots, x_{d}\right\}\end{array}$ & $\begin{array}{l}\bar{\partial}_{\omega} U:=\left(\partial_{\omega} u_{i j}\right)_{i j} \\
\omega \in\left\{t, x_{1}, \ldots, x_{d}\right\}\end{array}$ \\
\hline $\begin{array}{c}\text { higher } \\
\text { derivatives }\end{array}$ & $\begin{array}{c}\partial_{\omega}^{k} u \\
\omega \in\left\{t, x_{1}, \ldots, x_{d}\right\}\end{array}$ & $\begin{array}{l}\bar{\partial}_{\omega}^{k} U:=\left(\partial_{\omega}^{k} u_{i j}\right)_{i j} \\
\omega \in\left\{t, x_{1}, \ldots, x_{d}\right\}\end{array}$ \\
\hline Laplacian & $\Delta u:=\sum_{i=1}^{d} \partial_{x_{i}}^{2} u$ & $\bar{\Delta} U:=\sum_{i=1}^{d} \bar{\partial}_{x_{i}}^{2} U$ \\
\hline gradient & $\begin{array}{c}\nabla u(x):=\left(\partial_{x_{1}} u(x), \ldots, \partial_{x_{d}} u(x)\right)^{\top} \\
\nabla u(x) \in \mathbb{R}^{d}\end{array}$ & $\begin{array}{c}\bar{\nabla} U(x):=\left(\bar{\partial}_{x_{1}} U(x), \ldots, \bar{\partial}_{x_{d}} U(x)\right)^{\top} \\
\bar{\nabla} U(x) \in\left(\operatorname{Sym}_{n}(\mathbb{R})\right)^{d}\end{array}$ \\
\hline divergence & $\begin{array}{c}\operatorname{div}(a(x))^{\top}:=\sum_{i=1}^{d} \partial_{x_{i}} a_{i}(x) \\
a(x):=\left(a_{1}(x), \ldots, a_{d}(x)\right)\end{array}$ & $\begin{array}{c}\overline{\operatorname{div}}(A(x))^{\top}:=\sum_{i=1}^{d} \bar{\partial}_{x_{i}} A_{i}(x) \\
A(x):=\left(A_{1}(x), \ldots, A_{d}(x)\right)\end{array}$ \\
\hline length & $\begin{aligned}|w|_{p}:= & \sqrt[p]{\left|w_{1}\right|^{p}+\cdots+\left|w_{d}\right|^{p}} \\
& |w|_{p} \in[0,+\infty[\end{aligned}$ & $\begin{array}{c}|W|_{p}:=\sqrt[p]{\left|W_{1}\right|^{p}+\cdots+\left|W_{d}\right|^{p}} \\
|W|_{p} \in \operatorname{Sym}_{n}^{+}(\mathbb{R})\end{array}$ \\
\hline multiplication & $a \cdot b$ & $A^{\frac{1}{2}} B A^{\frac{1}{2}}$ \\
\hline
\end{tabular}


matrix field of orthogonal matrices, while $x \mapsto D(x)$ is a matrix field of diagonal matrices. In the sequel we will denote $n \times n$ - diagonal matrices with entries $\lambda_{1}, \ldots, \lambda_{n} \in \mathbb{R}$ from left to right simply by $\operatorname{diag}\left(\lambda_{i}\right) . O(n)$ stands for the matrix group of orthogonal $n \times n$-matrices. We will also assume that the matrix field $U(x)$ to be diagonalisable with $U=\left(u_{i, j}\right)_{i, j}=V^{\top} \operatorname{diag}\left(\lambda_{1}, \ldots, \lambda_{n}\right) V$, where $V \in O(n)$ and $\lambda_{1}, \ldots, \lambda_{n} \in \mathbb{R}$.

\section{Remarks 1:}

1. Functions of matrices. The definition of a function $h$ on $\operatorname{Sym}_{n}(\mathbb{R})$ is standard [12. As an important example, $|U|$ denotes the matrix-valued equivalent of the absolute value of a real number, $|U|=V^{\top} \operatorname{diag}\left(\left|\lambda_{1}\right|, \ldots,\left|\lambda_{n}\right|\right) V \in$ $\operatorname{Sym}_{n}^{+}(\mathbb{R})$, not to be confused with the determinant $\operatorname{det}(U)$ of $U$.

2. Partial derivatives. The componentwise definition of the partial derivative for matrix fields is a natural extension of the scalar case:

$$
\begin{aligned}
\bar{\partial}_{\omega} U\left(\omega_{0}\right) & =\lim _{h \rightarrow 0} \frac{1}{h}\left(U\left(\omega_{0}+h\right)-U\left(\omega_{0}\right)\right)=\left(\lim _{h \rightarrow 0} \frac{u_{i j}\left(\omega_{0}+h\right)-u_{i j}\left(\omega_{0}\right)}{h}\right)_{i, j} \\
& =\left(\partial_{\omega} u_{i j}\left(\omega_{0}\right)\right)_{i, j},
\end{aligned}
$$

where $\bar{\partial}_{\omega}$ stands for a spatial or temporal derivative. By iteration, higher order partial differential operators such as the Laplacian or other more sophisticated operators find their natural counterparts in the matrix-valued framework.

It is worth mentioning that for the operators $\bar{\partial}_{\omega}$ a product rule holds:

$$
\left.\bar{\partial}_{\omega}(A(x) \cdot B(x))=\left(\bar{\partial}_{\omega} A(x)\right) \cdot B(x)\right)+A(x) \cdot\left(\bar{\partial}_{\omega} B(x)\right) .
$$

Observe that positive definiteness in general is not preserved through derivation $\bar{\partial}_{\omega}$.

3. Generalized gradient of a matrix field. The definition of a generalised gradient is somewhat different from one that might be expected when viewing a matrix as a tensor (of second order). The rules of differential geometry would tell us that derivatives are tensors of third order. Instead, we adopt a more operator-algebraic point of view: The matrices are self-adjoint operators that can be added, multiplied with a scalar, and concatenated. Thus, they form an algebra, and we aim at consequently replacing the field $\mathbb{R}$ by the algebra $\operatorname{Sym}_{n}(\mathbb{R})$ in the scalar, that is, $\mathbb{R}$-based formulation of PDEs used in image processing. Hence, the generalised gradient $\bar{\nabla} U(x)$ at a voxel $x$ is regarded as an element of the module $\left(\operatorname{Sym}_{n}(\mathbb{R})\right)^{d}$ over $\operatorname{Sym}_{n}(\mathbb{R})$ in close analogy to the scalar setting where $\nabla u(x) \in \mathbb{R}^{d}$.

In the sequel we will call a mapping from $\mathbb{R}^{d}$ into $\left(\operatorname{Sym}_{n}(\mathbb{R})\right)^{d}$ a module field rather than a vector field.

4. Generalised divergence of the module field. The generalization of the divergence operator div acting on a vector field to an operator $\overline{\operatorname{div}}$ acting 
on a module field $A$ is straightforward, and is in accordance with the formal relation $\bar{\Delta} U=\overline{\operatorname{div}} \bar{\nabla} U=\bar{\nabla} \cdot \bar{\nabla} U$ known in its scalar form from standard vector analysis.

5. Generalised Length in $\left(\mathbf{S y m}_{n}(\mathbb{R})\right)^{d}$. Considering the formal definition in Table 1 the length of an element of a module field $A$ is close at hand. It results in a positive semidefinite matrix from $\operatorname{Sym}_{n}^{+}(\mathbb{R})$ the direct counterpart of a nonnegative real number as the length of a vector in $\mathbb{R}^{d}$.

6. Symmetrised Multiplication in $\mathbf{S y m}_{n}(\mathbb{R})$. The scalar TV-diffusion equation (11) requires the multiplication of the components of a vector (namely $\nabla u$ ) with a scalar (namely $\frac{1}{\|\nabla u\|}$ ). In the matrix-valued setting the components of $\bar{\nabla} U$, that is, $\bar{\partial}_{x_{i}} U, i=1, \ldots, d$, and (the inverse of) its generalised length $|\bar{\nabla} U|_{2}=:|\bar{\nabla} U|$ are symmetric matrices. However, the product of two symmetric matrices $A, B \in \operatorname{Sym}_{n}(\mathbb{R})$ is not symmetric unless the matrices commute. Among the numerous options to define a symmetrised matrix product we focus on one that is inspired from pre-conditioning of symmetric linear equation systems [12]. We define

$$
A \bullet B:=A^{\frac{1}{2}} B A^{\frac{1}{2}}
$$

as the symmetrised multiplication of symmetric matrices.

For the sake of future comparison we first consider the matrix-valued version of the linear diffusion equation on $\mathbb{R}^{d} \times[0, \infty[$ in the next section.

\section{Diffusion Equations for Matrix-Fields}

\subsection{Matrix-Valued Linear Diffusion}

The linear diffusion equation $\partial_{t} u=\sum_{i=1}^{d} \partial_{x_{i}} \partial_{x_{i}} u=\sum_{i=1}^{d} \partial_{x_{i} x_{i}} u=\bar{\Delta} u$ on $\mathbb{R}^{d} \times[0, \infty[$ is directly extended to the matrix valued setting:

$$
\bar{\partial}_{t} U=\sum_{i=1}^{d} \bar{\partial}_{x_{i}} \bar{\partial}_{x_{i}} U=\sum_{i=1}^{d} \bar{\partial}_{x_{i} x_{i}} U=\bar{\Delta} U
$$

with initial condition $U(x, 0)=F(x)$. The diffusion process described by this equation acts on each of the components of the matrix independently. It is proven in [11] that positive (semi-)definiteness of the initial matrix field $F$ is indeed bequeathed to $U$ for all times.

\subsection{Matrix-Valued Singular Diffusion Equations}

In Section 2, Remark 1, (6) we set $A \bullet B:=A^{\frac{1}{2}} B A^{\frac{1}{2}}$ for a symmetric multiplication of symmetric matrices. It is easily verified that this product is neither associative, nor commutative, and distributive only in the second argument. However, if $A$ is non-singular, the so-called signature $s=\left(s_{+}, s_{-}, s_{0}\right)$ of $B$ is 
preserved, where $s_{+}, s_{-}$, and $s_{0}$, stand for the number of positive, negative, and vanishing eigenvalues of $B$, respectively. This implies in particular that the positive definiteness of $B$ is preserved. Furthermore, for commuting matrices $A, B$ we have $A \bullet B=A \cdot B$. Another even more prominent candidate for a symmetrised multiplication would be the so-called Jordan product $A \bullet_{J} B:=\frac{1}{2}(A B+B A)$, which is neither associative nor distributive, but commutative. The reason we disregard it in this article lies in the fact that it does not preserve positive (semi-)definiteness as the following simple example shows:

$$
\left(\begin{array}{ll}
2 & 0 \\
0 & 0
\end{array}\right) \cdot J\left(\begin{array}{ll}
1 & 1 \\
1 & 1
\end{array}\right)=\frac{1}{2}\left(\left(\begin{array}{ll}
2 & 2 \\
0 & 0
\end{array}\right)+\left(\begin{array}{ll}
2 & 0 \\
2 & 0
\end{array}\right)\right)=\left(\begin{array}{ll}
2 & 1 \\
1 & 0
\end{array}\right) \text { but } \operatorname{det}\left(\begin{array}{ll}
2 & 1 \\
1 & 0
\end{array}\right)=-1 .
$$

Remark 2: It should be mentioned that the logarithmic multiplication introduced in [5] and given by $A \bullet_{L} B:=\exp (\log (A)+\log (B))$ is defined only for positive definite matrices. However, the matrix-valued PDE-based filtering proposed here requires the symmetric multiplication to be able to cope with at least one factor matrix being indefinite. Furthermore, matrix fields that are not necessarily positive semidefinite should also be within the reach of our PDE-based methods. Hence the logarithmic multiplication is not suitable for our purpose.

With these definitions we are now in the position to state the matrix-valued counterparts for the PDEs (11)-(3) mentioned above. For the sake of brevity we concentrate on the most general one, the self-snakes:

$$
\begin{aligned}
\partial_{t} u & =|\bar{\nabla} U| \bullet \operatorname{div}\left(\frac{g\left(|\bar{\nabla} U|^{2}\right)}{|\bar{\nabla} U|} \bullet \bar{\nabla} U\right) \\
& =\sqrt{|\bar{\nabla} U|} \cdot\left[\sum_{i=1}^{d} \bar{\partial}_{x_{i}}\left(\sqrt{\frac{g\left(|\bar{\nabla} U|^{2}\right)}{|\bar{\nabla} U|}} \cdot\left(\bar{\partial}_{x_{i}} U\right) \cdot \sqrt{\frac{g\left(|\bar{\nabla} U|^{2}\right)}{|\bar{\nabla} U|}}\right)\right] \cdot \sqrt{|\bar{\nabla} U|},
\end{aligned}
$$

where we used the notation

$$
\frac{g\left(|\bar{\nabla} U|^{2}\right)}{|\bar{\nabla} U|}:=g\left(|\bar{\nabla} U|^{2}\right) \cdot|\bar{\nabla} U|^{-1}=|\bar{\nabla} U|^{-1} \cdot g\left(|\bar{\nabla} U|^{2}\right)=|\bar{\nabla} U|^{-1} \bullet g\left(|\bar{\nabla} U|^{2}\right) \text {. }
$$

Specifying $g=1$ we regain the matrix-valued PDE for mean curvature motion of matrix fields, while neglecting the factor $|\bar{\nabla} U|$ and setting $g\left(s^{2}\right)=\frac{1}{|s|}$ in equation (5) produces the equation for BFB-diffusion, for instance.

\subsection{Matrix-Valued Signals}

In this section we investigate matrix-valued TV-related diffusion processes, mean curvature motion and self-snakes in the case of one space dimension. We restrict ourselves to the one-dimensional case $(d=1), U: \mathbb{R} \longrightarrow \operatorname{Sym}_{n}(\mathbb{R})$, since then simplifications occur. Only one spatial derivative appears, and the expressions containing the matrix $\bar{\partial}_{x}$ commute. Hence, in those expressions the symmetric multiplication "• collapses to ".", facilitating the analysis. The equation for the 
matrix-valued self-snakes in one space dimension simplifies to

$$
\bar{\partial}_{t} U=\left|\bar{\partial}_{x} U\right| \bullet \bar{\partial}_{x}\left(\frac{g\left(\left(\bar{\partial}_{x} U\right)^{2}\right)}{\left|\bar{\partial}_{x} U\right|} \cdot \bar{\partial}_{x} U\right) .
$$

However, even in this simplified setting this type of data exhibits directional (through eigenvectors) as well as shape information (through eigenvalues) which allows for the appearance of new phenomena. The partial derivative $\bar{\partial}_{x}$ of a signal $U$ of symmetric matrices results again in symmetric matrices, $\bar{\partial}_{x} U(x) \in$ $\operatorname{Sym}_{n}(\mathbb{R})$. Hence we have $\bar{\partial}_{x} U(x)=\tilde{V}^{\top}(x) \operatorname{diag}\left(\tilde{\lambda}_{i}(x)\right) \tilde{V}(x)$ with $\tilde{V}(x) \in O(n)$ for all $x \in \Omega$. We observe that $\frac{g\left(\left(\bar{\partial}_{x} U\right)^{2}\right)}{\left|\bar{\partial}_{x} U\right|}$ is also diagonalised by $\tilde{V}$,

$$
\frac{g\left(\left(\bar{\partial}_{x} U\right)^{2}\right)}{\left|\bar{\partial}_{x} U\right|}=\tilde{V}^{\top} \operatorname{diag}\left(\frac{g\left(\tilde{\lambda}_{i}^{2}\right)}{\left|\tilde{\lambda}_{i}\right|}\right) \tilde{V},
$$

and introducing the abbreviation $h\left(s^{2}\right):=\frac{g\left(s^{2}\right)}{\sqrt{s^{2}}}$ it follows that $h\left(\left(\bar{\partial}_{x} U\right)^{2}\right) \cdot \bar{\partial}_{x} U=$ $\tilde{V}^{\top} \operatorname{diag}\left(h\left({\tilde{\lambda_{i}}}^{2}\right) \cdot \tilde{\lambda}_{i}\right) \quad \tilde{V}$. We introduce a flux function $\Phi$ by $\Phi(s):=s \cdot h\left(s^{2}\right)$ which gives $\frac{d \Phi}{d s}(s)=\Phi^{\prime}(s)=2 s^{2} h^{\prime}\left(s^{2}\right)+h\left(s^{2}\right)$ at least for $s \neq 0$. In order to treat the singularity at $s=0$ it is customary to regularise $h$ in one way or the other to make $h$ differentiable in $[0,+\infty[$. Keeping numerical issues in mind we also adopt this point of view, rather than interpreting the derivatives in the following calculations in the distributional sense. The product rule for matrixvalued functions and incorporating $\Phi$ then yields, if we suppress the explicit dependence of $V$ and $\lambda_{i}$ on $x$ notationally, the following matrix-valued version of the self-snakes equation

$$
\begin{gathered}
\bar{\partial}_{t} U=\left|\bar{\partial}_{x} U\right| \bullet\left(\bar{\partial}_{x} \tilde{V} \operatorname{diag}\left(h\left({\tilde{\lambda_{i}}}^{2}\right) \cdot \tilde{\lambda}_{i}\right) \tilde{V}^{\top}+\tilde{V} \operatorname{diag}\left(h\left({\tilde{\lambda_{i}}}^{2}\right) \cdot \tilde{\lambda}_{i}\right) \bar{\partial}_{x} \tilde{V}^{\top}\right. \\
\left.\left.+\tilde{V} \operatorname{diag}\left(\Phi^{\prime}\left(\tilde{\lambda}_{i}\right) \cdot \partial_{x} \tilde{\lambda}_{i}\right]\right) \tilde{V}^{\top}\right)
\end{gathered}
$$

We infer that the matrix-valued data allow for a new phenomenon: unlike in the scalar setting, a matrix carries directional information conveyed through the eigenvectors as well as shape information mediated via eigenvalues. The evolution process described in (6) and (7) displays a coupling between shape and directional information by virtue of the simultaneous occurrence of terms containing $\bar{\partial}_{x} \tilde{V}(x)$ in (6) and $\partial_{x} \tilde{\lambda}(x)$ in (7). Clearly there is no equivalent for this in the scalar setting.

\section{Matrix-Valued Numerical Schemes}

In the previous sections the guideline to infer matrix-valued PDEs from scalar ones was, roughly speaking, analogy by making a transition from the real field

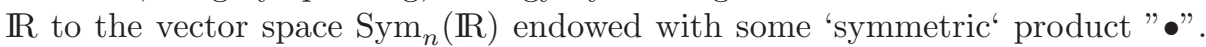


We follow this guideline also in the issue of numerical schemes for matrix-valued PDEs.

For the sake of brevity we restrict ourselves to the TV-type diffusion, which means $h\left(s^{2}\right)=\frac{1}{\sqrt{s^{2}}}\left(\right.$ or in its regularised form $h\left(s^{2}\right)=\frac{1}{\sqrt{\varepsilon^{2}+s^{2}}}$ with $0 \leq \varepsilon \ll 1$ ) and two space dimensions $(d=2)$. The necessary extensions to self-snakes in dimensions $d \geq 3$ are immediate. A possible space-discrete scheme for the scalar $\mathrm{TV}$-diffusion can be cast into the form

$$
\begin{aligned}
\frac{d u(i, j)}{d t} & =\frac{1}{\tau_{1}}\left(h\left(i+\frac{1}{2}, j\right) \cdot \frac{u(i+1, j)-u(i, j)}{\tau_{1}}-h\left(i-\frac{1}{2}, j\right) \cdot \frac{u(i, j)-u(i-1, j)}{\tau_{1}}\right) \\
& +\frac{1}{\tau_{2}}\left(h\left(i, j+\frac{1}{2}\right) \cdot \frac{u(i, j+1)-u(i, j)}{\tau_{2}}-h\left(i, j-\frac{1}{2}\right) \cdot \frac{u(i, j)-u(i, j-1)}{\tau_{2}}\right),
\end{aligned}
$$

where $h(i, j)$ and $u(i, j)$ are samples of the (regularised) diffusivity $h$ and of $u$ at pixel $(i, j)$ and, for example, $h\left(i \pm \frac{1}{2}, j\right):=\frac{h(i \pm 1, j)+h(i, j)}{2}$. According to our preparations in Section 2 its matrix-valued extension to solve the TV-diffusion equation in the matrix setting reads

$$
\begin{aligned}
\frac{d U(i, j)}{d t} & =\frac{1}{h_{1}}\left(H\left(i+\frac{1}{2}, j\right) \bullet \frac{U(i+1, j)-U(i, j)}{h_{1}}-H\left(i-\frac{1}{2}, j\right) \bullet \frac{U(i, j)-U(i-1, j)}{h_{1}}\right) \\
& +\frac{1}{h_{2}}\left(H\left(i, j+\frac{1}{2}\right) \bullet \frac{U(i, j+1)-U(i, j)}{h_{2}}-H\left(i, j-\frac{1}{2}\right) \bullet \frac{U(i, j)-U(i, j-1)}{h_{2}}\right) .
\end{aligned}
$$

The arithmetic mean $H\left(i \pm \frac{1}{2}, j\right):=\frac{H(i \pm 1, j)+H(i, j)}{2} \in \operatorname{Sym}_{n}(\mathbb{R})$ approximates the diffusivity $H\left(|\bar{\nabla} U|^{2}\right)$ between the pixels $(i \pm 1, j)$ and $(i, j)$. However, for the numerical treatment of MCM and self-snakes the usage of the properly defined harmonic mean instead of the arithmetic mean is advised. In the scalar setting this was already observed and put to work in [18].

\section{Experiments}

In our experiments we used a 3-D DT-MRI data set of a human head consisting of a $128 \times 128 \times 38$-field of positive definite matrices. The data are represented as ellipsoids via the level sets of the quadratic form $\left\{x^{\top} A^{-2} x=\right.$ const. : $\left.x \in \mathbb{R}^{3}\right\}$ associated with a matrix $A \in \operatorname{Sym}^{+}(3)$. By using $A^{-2}$ the length of the semi-axes of the ellipsoid correspond directly with the three eigenvalues of the matrix. However, for a better judgement of the denoising qualities of the smoothing processes we utilise also artificial data sets.

In Figure 1 below we compare the results of matrix-valued TV-and BFBdiffusion. The noise is removed while the edge is preserved, in very good agreement with the well-known denoising properties of their scalar predecessors.

Another set of artificial data, depicted in Figure 2, is used to demonstrate exemplarily the denoising capabilities of matrix-valued self-snakes, see Figure 3.Figure 4 juxtaposes matrix-valued linear diffusion, and smoothing with MCM and selfsnakes. The smoothing as well as the convexifying and shrinking of image objects to circular structures known as features of scalar mean curvature motion 

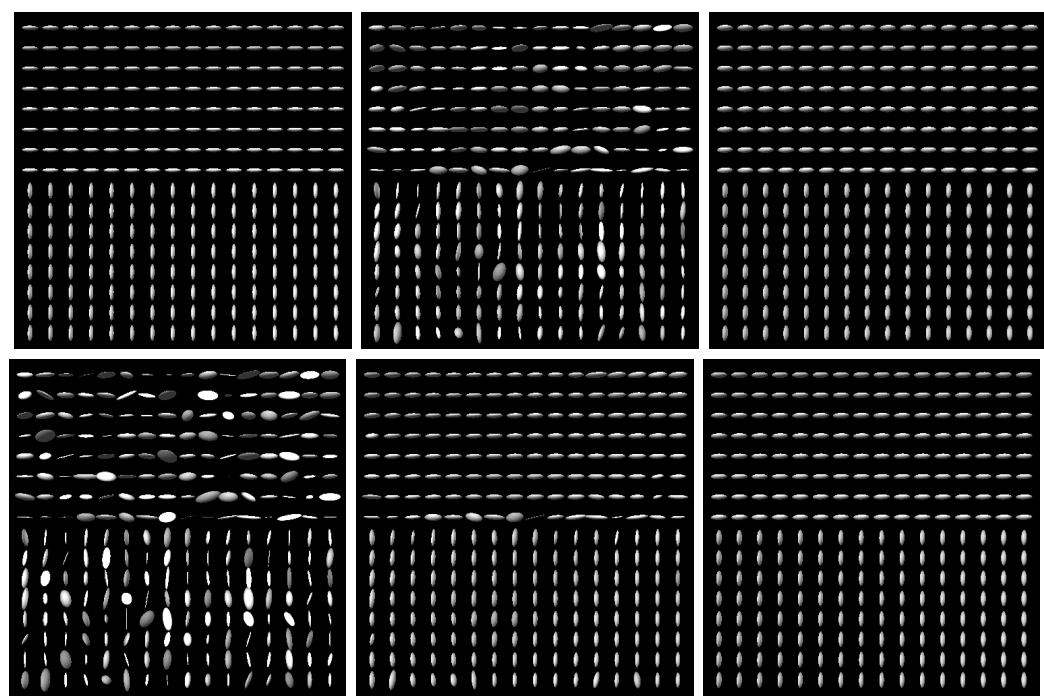

Fig. 1. (a) Top row, from left to right: Original matrix field. TV-diffusion on the noisy image after $t=5$, and $t=100$. (b) Bottom row, from left to right: Original polluted additively with a random matrix field $R$. The eigenvalues of $R$ stem from a Gaussian distribution with vanishing mean and standard deviation 100, its normalised eigenvectors have uniform spatial distribution. Then BFB-diffusion on the noisy image after $t=0.5$, and $t=10$.
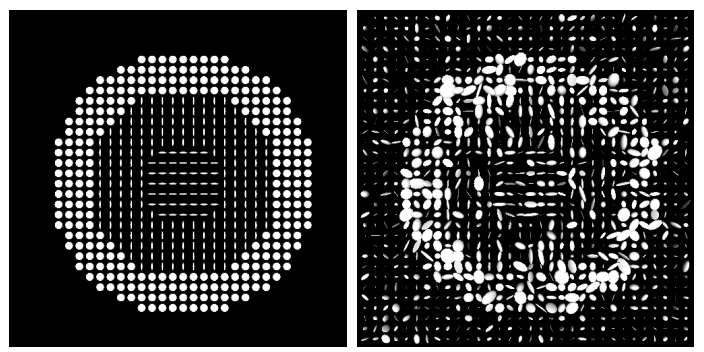

Fig. 2. Left: Original matrix field. Right: Original polluted additively with a random matrix field $R$ as in Figure 1
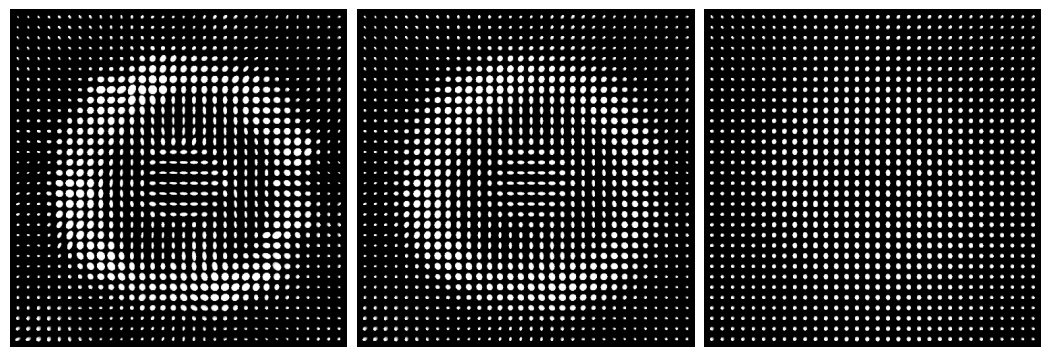

Fig. 3. From left to right: Filtering results for the polluted image of Figure 2 with self-snakes $(\lambda=2000)$ after $t=5, t=10$, and $t=100$ 

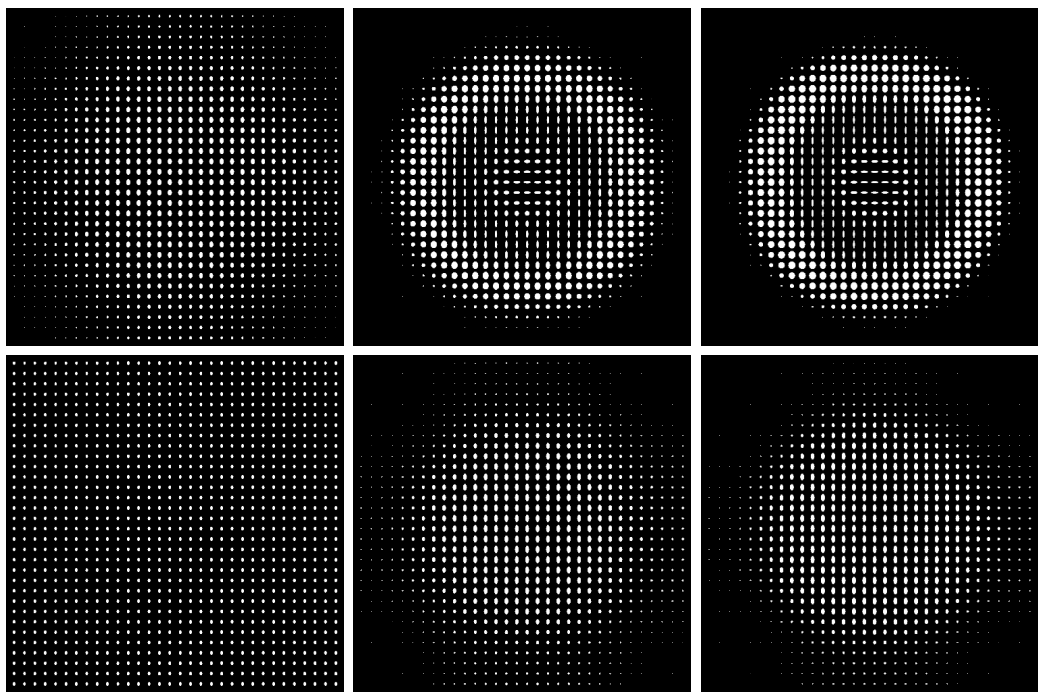

Fig. 4. Smoothing of image (a) in Figure 2 (a) First column, top to bottom: Linear Diffusion. Stopping times $t=10$, and $t=100$. (b) Second column, top to bottom: Mean curvature motion. Stopping times $t=10$, and $t=100$. (c) Third column, top to bottom: Self-snakes with $\lambda=2000$. Stopping times $t=10$, and $t=100$.
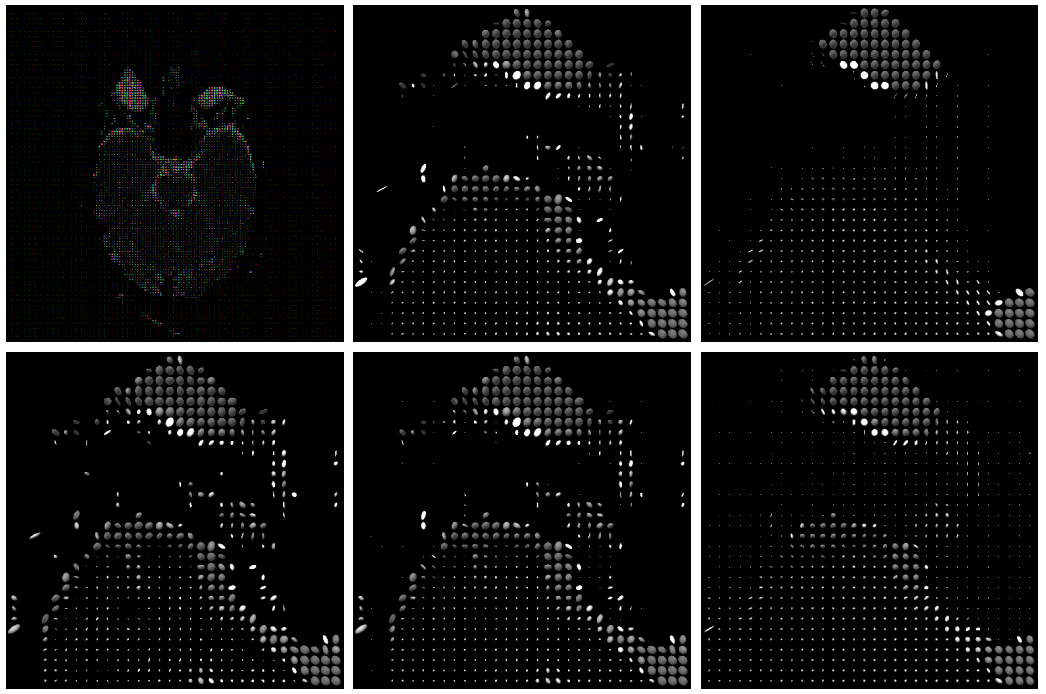

Fig. 5. (a) Top row, from left to right: Original: 2D slice of a 3D DT-MRI image of a human brain. Smoothing with self-snakes $(\lambda=2000)$ after $t=5$, and $t=50$. (b) Bottom row, from left to right: Enlarged section of the original. Smoothing with TV-diffusion after $t=5$, and $t=50$. 
and self-snakes are clearly discernable in our matrix-valued setting. Finally, in Figure 5the smoothing and enhancing properties of matrix-valued self-snakes and TV-diffusion are juxtaposed while acting on a 2-D slice of a real 3-D DT-MRI data set. The matrix-valued extensions inherit the filtering capabilities of their scalar counterparts.

It is worth mentioning that the results are in good agreement with the results in [11] and [16]. However, the framework presented here is generic, hence more general, and does not rely on any notion of a potentially parameter-steered structure tensor.

\section{Conclusion}

In this article we have presented a novel and generic framework for the extension of singular PDEs to symmetric matrix fields in any spatial dimension. We focused on the extension of scalar TV/BFB-diffusion, mean curvature motion, and self-snakes as leading examples. The approach takes an operator-algebraic point of view and ensures appropriate channel interaction without the use of a structure tensor. Experiments on positive semidefinite DT-MRI and artificial data illustrate that the matrix-valued methods inherit desirable characteristic properties of their scalar valued predecessors, e.g. very good denoising capabilities combined with feature preserving qualities, and the absence of tuning parameters. In future work we will investigate how this framework can help to extend other scalar PDEs and more sophisticated numerical solution concepts in image processing to the matrix-valued setting.

\section{References}

1. L. Alvarez, F. Guichard, P.-L. Lions, and J.-M. Morel. Axioms and fundamental equations in image processing. Archive for Rational Mechanics and Analysis, 123:199-257, 1993.

2. L. Alvarez, P.-L. Lions, and J.-M. Morel. Image selective smoothing and edge detection by nonlinear diffusion. II. SIAM Journal on Numerical Analysis, 29: 845-866, 1992.

3. F. Andreu, C. Ballester, V. Caselles, and J. M. Mazón. Minimizing total variation flow. Differential and Integral Equations, 14(3):321-360, March 2001.

4. F. Andreu, V. Caselles, J. I. Diaz, and J. M. Mazón. Qualitative properties of the total variation flow. Journal of Functional Analysis, 188(2):516-547, February 2002.

5. V. Arsigny, P. F., X. Pennec, and N. Ayache. Fast and simple calculus on tensors in the log-Euclidean framework. In J. Duncan and G. Gerig, editors, Medical Image Computing and Computer-Assisted Intervention - MICCAI 2005, Part I, volume 3749 of LNCS, pages 115-122. Springer, 2005.

6. G. Bellettini, V. Caselles, and M. Novaga. The total variation flow in $R^{N}$. Journal of Differential Equations, 184(2):475-525, 2002.

7. T. Brox, J. Weickert, B. Burgeth, and P. Mrázek. Nonlinear structure tensors. Image and Vision Computing, 24(1):41-55, 2006. 
8. B. Burgeth, A. Bruhn, S. Didas, J. Weickert, and M. Welk. Morphology for matrixdata: Ordering versus PDE-based approach. Image and Vision Computing, 2006.

9. C. Chefd'Hotel, D. Tschumperlé, R. Deriche, and O. Faugeras. Constrained flows of matrix-valued functions: Application to diffusion tensor regularization. In A. Heyden, G. Sparr, M. Nielsen, and P. Johansen, editors, Computer Vision - ECCV 2002, volume 2350 of Lecture Notes in Computer Science, pages 251-265. Springer, Berlin, 2002.

10. F. Dibos and G. Koepfler. Total variation minimization by the Fast Level Sets Transform. In Proc. First IEEE Workshop on Variational and Level Set Methods in Computer Vision, pages 145-152, Vancouver, Canada, July 2001. IEEE Computer Society Press.

11. C. Feddern, J. Weickert, B. Burgeth, and M. Welk. Curvature-driven PDE methods for matrix-valued images. International Journal of Computer Vision, 69(1):91-103, August 2006.

12. R. A. Horn and C. R. Johnson. Matrix Analysis. Cambridge University Press, Cambridge, UK, 1990.

13. S. L. Keeling and R. Stollberger. Nonlinear anisotropic diffusion filters for wide range edge sharpening. Inverse Problems, 18:175-190, January 2002.

14. C. Pierpaoli, P. Jezzard, P. J. Basser, A. Barnett, and G. Di Chiro. Diffusion tensor MR imaging of the human brain. Radiology, 201(3):637-648, December 1996.

15. G. Sapiro. Geometric Partial Differential Equations and Image Analysis. Cambridge University Press, Cambridge, UK, 2001.

16. T. Schultz, B. Burgeth, and J. Weickert. Flexible segmentation and smoothing of DT-MRI fields through a customizable structure tensor. In Proceedings of the International Symposium on Visual Computing, Lecture Notes in Computer Science. Springer, Berlin, 2007.

17. V. I. Tsurkov. An analytical model of edge protection under noise suppression by anisotropic diffusion. Journal of Computer and Systems Sciences International, 39(3):437-440, 2000.

18. J. Weickert. Applications of nonlinear diffusion in image processing and computer vision. Acta Mathematica Universitatis Comenianae, 70(1):33-50, 2001.

19. J. Weickert and T. Brox. Diffusion and regularization of vector- and matrixvalued images. In M. Z. Nashed and O. Scherzer, editors, Inverse Problems, Image Analysis, and Medical Imaging, volume 313 of Contemporary Mathematics, pages 251-268. AMS, Providence, 2002.

20. J. Weickert and H. Hagen, editors. Visualization and Processing of Tensor Fields. Springer, Berlin, 2006. 